\title{
Top Quark Pair Production in Association with a Jet with NLO QCD Off-Shell Effects at the Large Hadron Collider
}

\author{
G. Bevilacqua ${ }^{a}$, H. B. Hartanto $^{b}$, M. $\operatorname{Kraus}^{b}$ And M. Worek ${ }^{b}$ \\ ${ }^{a}$ INFN, Laboratori Nazionali di Frascati, Via E. Fermi 40, I-00044 Frascati, Italy \\ ${ }^{b}$ Institut für Theoretische Teilchenphysik und Kosmologie, \\ RWTH Aachen University, D-52056 Aachen, Germany
}

\begin{abstract}
We present a complete description of top quark pair production in association with a jet in the dilepton channel. Our calculation is accurate to next-to-leading order in QCD (NLO) and includes all non-resonant diagrams, interferences and off-shell effects of the top quark. Moreover, non-resonant and off-shell effects due to the finite $\mathrm{W}$ gauge boson width are taken into account. This calculation constitutes the first fully realistic NLO computation for top quark pair production with a final state jet in hadronic collisions. Numerical results for differential distributions as well as total cross sections are presented for the Large Hadron Collider (LHC) at $8 \mathrm{TeV}$. With our inclusive cuts, NLO predictions reduce the unphysical scale dependence by more than a factor of 3 and lower the total rate by about $13 \%$ compared to leading order QCD (LO) predictions. In addition, the size of the top quark off-shell effects is estimated to be below $2 \%$.
\end{abstract}

Introduction: Top quark studies are currently driven by the LHC experiments. An exploration of top quark production and decay dynamics is among the main physics goals of ATLAS and CMS. Besides the determination of the top quark mass, key measurements at the LHC include the total cross section, kinematic distributions, spin correlations and top quark couplings to the $W$ and $Z$ bosons, photon and the Standard Model (SM) Higgs boson. Searches for rare top quark decays to probe physics beyond the SM also play a prominent role in research programs of both experimental collaborations. The top quark, however, is an extremely short lived resonance and only its decay products can be detected experimentally. In general, for comparison with data, theoretical predictions must include top quark decays. In the SM, a top quark decays almost exclusively to a $W$ boson and a $b$ quark. The experimentally cleanest top quark decay channel comprises leptonic $W$ gauge boson decays. The signature for this channel consists of two well isolated and oppositely charged leptons with high transverse momenta, $p_{T}$, large missing $p_{T}$ from invisible neutrinos and two jets, which originate from $b$ quarks. Due to the large collision energy at the LHC, $t \bar{t}$ pairs are abundantly produced with large $p_{T}$, hence, the probability for the top quark to radiate gluons is enough to make the $t \bar{t} j$ final state measurable with high statistics. In fact, for $p_{T j}>40 \mathrm{GeV}$, about half of the $t \bar{t}$ events are expected to be accompanied by an additional hard jet. The correct description of $t \bar{t} j$ production is, therefore, essential to study the top quark pair production at the LHC. For example, $t \bar{t} j$ can be employed in the measurement of the top quark mass by studying normalized differential distribution cross section with respect to its invariant mass [1]. Moreover, $t \bar{t} j$ constitutes an important background to pro- cesses with multijet final states. The most prominent being the SM Higgs boson production in the vector boson fusion with the following decay chain $H \rightarrow W^{+} W^{-} \rightarrow \ell^{+} \nu_{\ell} \ell^{-} \bar{\nu}_{\ell}[2,3]$. The $t \bar{t} j$ production plays a very important role in searches for physics beyond the SM. For example, it is one of the main backgrounds to processes such as supersymmetric particle production. Here, depending on the specific model, typical signals also include jets, charged leptons, and missing $p_{T}$ due to the escaping lightest supersymmetric particle [4]. Anomalous production of additional jets accompanying a $t \bar{t}$ pair could also be a sign of new physics beyond the SM [5].

The NLO corrections to $t \bar{t} j$ with stable top quarks have been first calculated in [6, 7]. Afterwards, LO top quark decays in the narrow width approximation (NWA) have been included [8]. Subsequently, NLO top quark decays in the NWA, including $t \rightarrow W b j$, have been added consistently [9]. A different path has been taken in [10 12], where matching to parton shower programs has been worked out. In this case, however, NLO corrections to the $t \bar{t} j$ production with stable top quarks have only been taken into account, while top quark decays, if included, have been modeled within parton shower frameworks.

In this Letter, we present a different approach. We drop altogether the approximation that top quarks are only produced on-shell, and concentrate on the fully realistic final state $p p \rightarrow e^{+} \nu_{e} \mu^{-} \bar{\nu}_{\mu} b \bar{b} j+X$. We consistently take into account resonant and non-resonant top quark contributions and all interference effects among them. In addition, non-resonant and off-shell effects due to the finite $W$ gauge boson width are included. Due to their insignificance we neglect flavor mixing as well as contributions from the suppressed initial bottom quark contributions. A few examples 

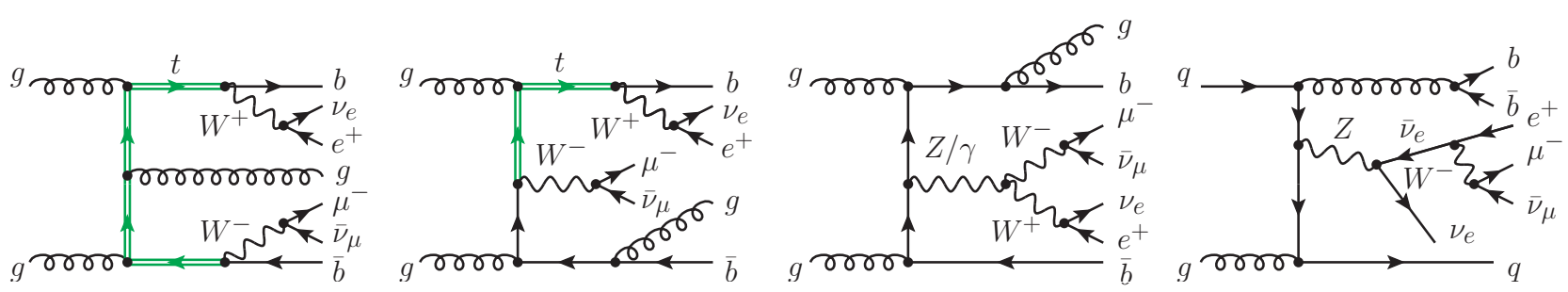

FIG. 1. Representative Feynman diagrams, involving two (first diagram), one (second diagram) and no top quark resonances (third diagram), contributing to the leading order $p p \rightarrow e^{+} \nu_{e} \mu^{-} \bar{\nu}_{\mu} b \bar{b} j$ process at $\mathcal{O}\left(\alpha_{s}^{3} \alpha^{4}\right)$. The last diagram with a single $W$ boson resonance contributes to the off-shell effects of the $W$ gauge boson.

of Feynman diagrams contributing to the leading order process at $\mathcal{O}\left(\alpha_{s}^{3} \alpha^{4}\right)$ are presented in Figure 1. We stress here that contributions of the order $O\left(\alpha_{s} \alpha^{6}\right)$ have not been included in our calculations. Full offshell top quark effects at NLO have already been considered in the literature for a simpler process, i.e. top quark pair production, first in [13, [14], and subsequently in 15 18]. Quite recently, a first attempt to go beyond the NWA for a $2 \rightarrow 5$ processes has been undertaken in [19], where NLO corrections to $p p \rightarrow e^{+} \nu_{e} \mu^{-} \bar{\nu}_{\mu} b \bar{b} H$ have been considered.

Calculation: NLO QCD corrections to $p p \rightarrow$ $e^{+} \nu_{e} \mu^{-} \bar{\nu}_{\mu} b \bar{b} j$ have been calculated with the HeLACNLO Monte Carlo program [20]. This is the first such computation with five final states (the decay products of the $W$ 's are not counted, because they do not couple to color charged states) carried out within this framework. We compute the virtual corrections in the 't Hooft-Veltman version of the dimensional regularisation using HelaC-1Loop 21] and CutTools 22], which are based on the Ossola-Papadopoulos-Pittau (OPP) reduction technique 23 25]. The most complicated one-loop diagrams in our calculations are heptagons. A number of optimizations have been devised in the algorithm of HELAC-1LOOP for the selection of loop topologies, which discard in advance all possibilities that are not compatible with the SM. This allowed us to substantially reduce the generation time. The process under consideration requires a special treatment of unstable top quarks, which is achieved within the complex mass scheme [26, 27]. At the one loop level the appearance of a non-zero top quark width in the propagator requires the evaluation of scalar integrals with complex masses, which is supported by the OnELOOP program, used for the evaluation of the integrals [28]. For consistency, mass renormalization for the top quark is also done by applying the complex mass scheme in the well known on-shell mass counter term. The preservation of gauge symmetries by this approach is explicitly checked by studying Ward identities up to the one loop level. Reweighting tech- niques, helicity and color sampling methods are additionally used in order to optimize the performance of our system. The singularities from soft or collinear parton emissions are isolated via subtraction methods for NLO QCD calculations. Specifically, two independent subtraction schemes are employed: the commonly used Catani-Seymour dipole subtraction 29 31, and a fairly new Nagy-Soper subtraction scheme 32], both implemented in the Helac-Dipoles software 31. The implementation consists of a phase space integrator of subtracted real radiation and integrated subtraction terms for massless and massive cases. The phase space integration is performed with the multichannel Monte Carlo generator Phegas [33] and KALEU [34]. In the latter case, dedicated additional channels for each subtraction term have been added for both subtraction schemes to improve the convergence of the phase space integrals for the subtracted real contribution. Let us also note, that we have implemented a new option in HELAC-NLO for automatically selecting the desired perturbative order in $\alpha_{s}$ and $\alpha$, preserving at the same time the structure and the advantages of the Dyson-Schwinger recursive approach for the construction of the amplitude. This modification is particularly useful in the current project, since we are interested in mixed contributions, i.e. $\mathcal{O}\left(\alpha_{s}^{3} \alpha^{4}\right)$ at $\mathrm{LO}$ and $\mathcal{O}\left(\alpha_{s}^{4} \alpha^{4}\right)$ at NLO.

Phenomenological Application: In the following we present our numerical results for $p p \rightarrow$ $e^{+} \nu_{e} \mu^{-} \bar{\nu}_{\mu} b \bar{b} j+X$ at the LHC at the center-of-mass energy of $\sqrt{s}=8 \mathrm{TeV}$. Decays of the weak bosons to different lepton generations are considered, to avoid virtual photon singularities arising from $\gamma \rightarrow \ell^{+} \ell^{-}$decays. These effects are at the level of $0.5 \%$, as checked by an explicit LO calculation. The SM parameters are set to

$$
\begin{array}{ll}
G_{\mathrm{F}}=1.16637 \cdot 10^{-5} \mathrm{GeV}^{-2}, & m_{\mathrm{t}}=173.3 \mathrm{GeV}, \\
m_{\mathrm{W}}=80.399 \mathrm{GeV}, & \Gamma_{\mathrm{W}}=2.09974 \mathrm{GeV}, \\
m_{\mathrm{Z}}=91.1876 \mathrm{GeV}, & \Gamma_{\mathrm{Z}}=2.50966 \mathrm{GeV},
\end{array}
$$




$$
\Gamma_{\mathrm{t}}^{\mathrm{LO}}=1.48132 \mathrm{GeV}, \Gamma_{\mathrm{t}}^{\mathrm{NLO}}=1.3542 \mathrm{GeV} .
$$

The top quark width has been calculated according to [35]. We use the MSTW2008 set of parton distribution functions (PDFs) [36], i.e. MSTW2008lo68cl PDFs with a 1-loop running $\alpha_{s}$ at LO and MSTW2008nlo68cl with a 2-loop running $\alpha_{s}$ at NLO. All light quarks including $b$ quarks, as well as leptons, are treated as massless. The suppressed contribution from $b$ quarks in the initial state is neglected. When considering the total cross section at LO this contribution amounts only to $0.8 \%$ of the total cross section. The renormalization and factorization scale is set to a common value $\mu_{\mathrm{R}}=\mu_{\mathrm{F}}=\mu_{0}=m_{\mathrm{t}}$. Let us notice that while evaluating $\Gamma_{\mathrm{t}}^{\mathrm{NLO}}$ the value of $\alpha_{s}$ at the scale $m_{\mathrm{t}}$ has been calculated from $\alpha_{s}\left(m_{\mathrm{Z}}\right)=0.118$. However, the $\alpha_{s}\left(m_{\mathrm{t}}\right)$ used within HELAC-NLO is obtained from the NLO MSTW2008 set that assumes $\alpha_{s}\left(m_{\mathrm{Z}}\right)=0.12018$. As a consequence, the corresponding $\alpha_{s}\left(m_{\mathrm{t}}\right)$ has a slightly different value. Should we use this value in the calculation of the top quark width, we would rather get $\Gamma_{\mathrm{t}}^{\mathrm{NLO}}=1.35207 \mathrm{GeV}$. The difference with respect to our value is at the level of one permille only, therefore, completely negligible for our NLO QCD results. Jets are defined out of partons with pseudorapidity $|\eta|<5$ by the anti- $k_{\mathrm{T}}$ jet algorithm [37] with the separation parameter $R=0.5$. We require exactly two $b$-jets, at least one light jet, two charged leptons and missing $p_{T}$. Final states have to fulfill the following kinematical requirements

$$
\begin{array}{ll}
p_{T \ell}>30 \mathrm{GeV}, & p_{T j}>40 \mathrm{GeV}, \\
p_{T}^{\text {miss }}>40 \mathrm{GeV}, & \Delta R_{j j}>0.5, \\
\Delta R_{\ell \ell}>0.4, & \Delta R_{\ell j}>0.4, \\
\left|y_{\ell}\right|<2.5, & \left|y_{j}\right|<2.5,
\end{array}
$$

where $\ell$ stands for $\mu^{-}, e^{+}$and $j$ corresponds to lightand $b$-jets. Results for the total cross sections are

$$
\begin{aligned}
\sigma_{\mathrm{HELAC-NLO}}^{\mathrm{LO}} & =183.1_{-64.2(35 \%)}^{+112.2(61 \%)} \mathrm{fb}, \\
\sigma_{\mathrm{HELAC-NLO}}^{\mathrm{NLO}} & =159.7_{-7.9(5 \%)}^{-33.1(21 \%)} \mathrm{fb} .
\end{aligned}
$$

At the central scale, the full $p p$ cross section receives negative and moderate NLO corrections of $13 \%$. Theoretical uncertainties, associated with neglected higher order terms in the perturbative expansion, have been estimated by varying the renormalization and factorization scales in $\alpha_{s}$ and PDFs, up and down by a factor of 2 around the central scale of the process, i.e. $\mu_{0}$. The scale uncertainties are evaluated to be $61 \%$ (48\% after symmetrization) at LO and $21 \%(13 \%$ after symmetrization) at NLO. Thus, a reduction of the theoretical error by a factor of 3 is observed. The

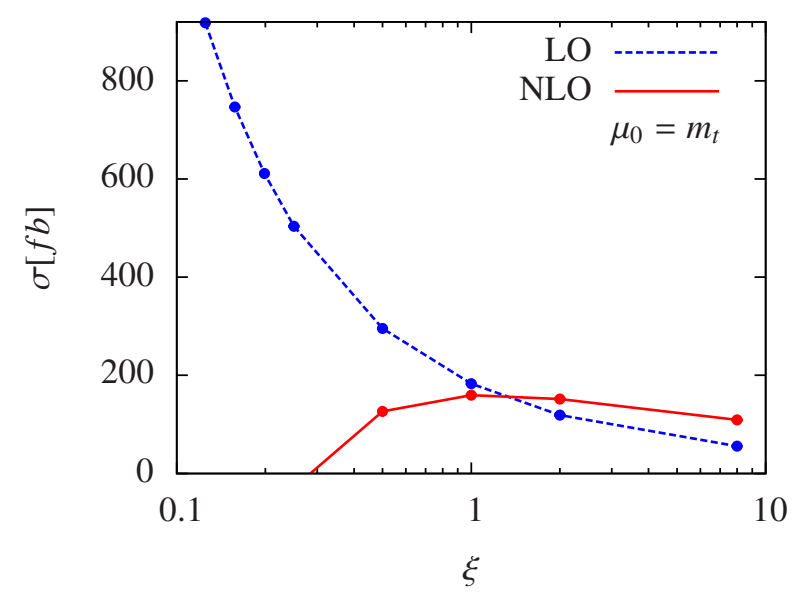

FIG. 2. Scale dependence of the LO and NLO cross sections for the $p p \rightarrow e^{+} \nu_{e} \mu^{-} \bar{\nu}_{\mu} b \bar{b} j+X$ process at the $L H C$ for $\sqrt{s}=8 \mathrm{TeV}$. The scale is set to a common value $\mu_{\mathrm{R}}=\mu_{\mathrm{F}}=\xi \mu_{0}$, where $\mu_{0}=m_{\mathrm{t}}$.

graphical presentation of the behavior of $\mathrm{LO}$ and NLO cross sections upon varying the scale by a factor of $\xi$ with $\xi \in\{0.125, \ldots, 8\}$ is shown in Figure 2.

In the next step, the size of the non-factorizable corrections for our setup is assessed. To that end, the full result has been compared with the result in the NWA, which has been obtained by rescaling the $t \rightarrow W b$ coupling and $\Gamma_{\mathrm{t}}$ by a small factor to mimic the limit $\Gamma_{\mathrm{t}} \rightarrow 0$. Finite top quark width effects change the cross section by less than $1 \%(2 \%)$ at LO (NLO), which is consistent with the expected uncertainty of the NWA, i.e. of the order of $\mathcal{O}\left(\Gamma_{\mathrm{t}} / m_{\mathrm{t}}\right)$. We have also calculated the NLO cross section with a setup from Ref. [9], where NLO QCD corrections in the NWA have been evaluated for the $p p \rightarrow e^{+} \nu_{e} e^{-} \bar{\nu}_{e} b \bar{b} j$ final state. Instead of using the top quark width from [9] we have calculated it afresh to account for the offshell effects of the $W$ gauge boson and obtained $\Gamma_{\mathrm{t}}=$ $1.31844 \mathrm{GeV}$. In addition, we have included bottom quark contributions in the initial state and required at least two $b$-jets in the final state. Our finding is $\sigma_{\text {HELAC-NLO }}^{\text {NLO }}=(275.5 \pm 0.6) \mathrm{fb}$. Comparing to the result from [9] we observe a $4.5 \%$ difference, which is of the order of the NWA accuracy for the top quark and the $W$ gauge boson. However, further investigation of the sources of the discrepancy would be desirable. We leave it for future study.

Representative differential distributions are presented in Figure 3 We exhibit the transverse momentum of the hardest (in $p_{T}$ ) light jet, $p_{T j_{1}}$, the separation between charged leptons in the rapidity azimuthal angle plane, $\Delta R_{e^{+} \mu^{-}}$, and the minimal invariant mass of the positron and $b$-jet, $M_{b e^{+}}$. The 

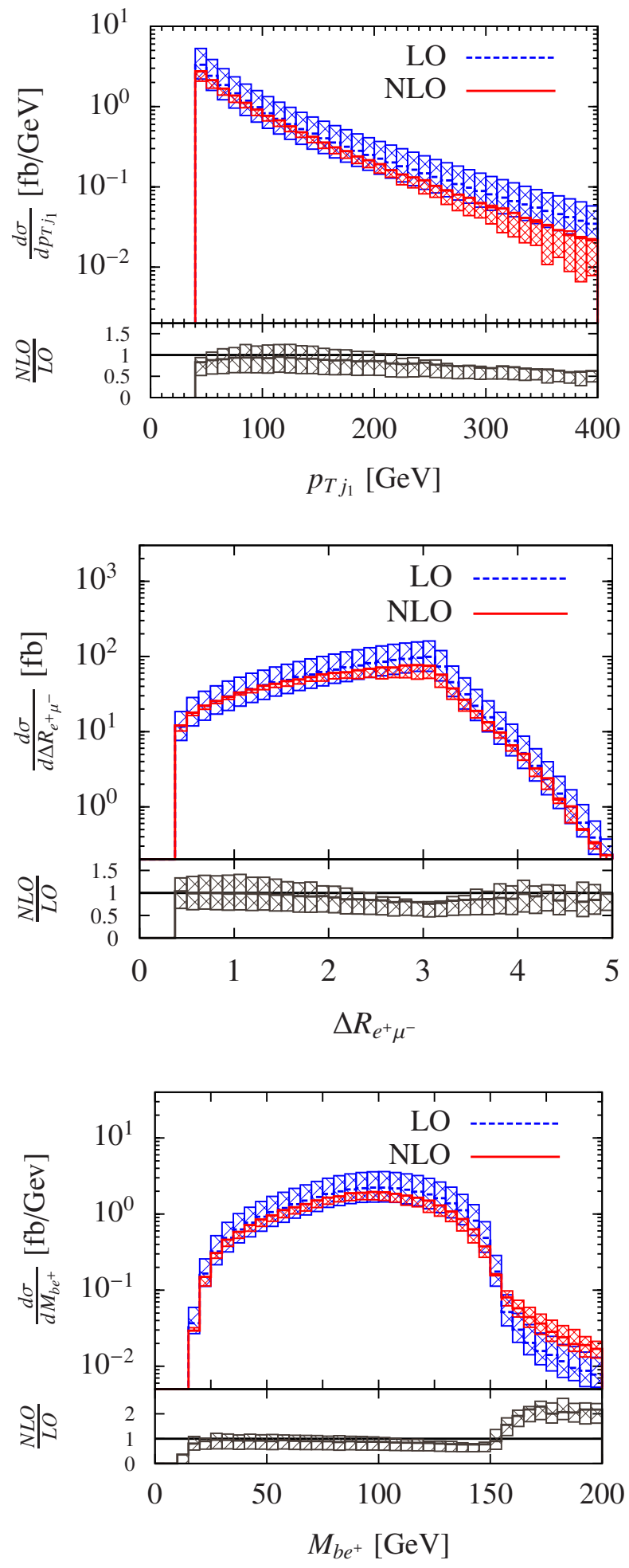

FIG. 3. Transverse momentum of the hardest light jet, $\Delta R_{e^{+} \mu^{-}}$and minimal $M_{b^{+}}$for $p p \rightarrow e^{+} \nu_{e} \mu^{-} \bar{\nu}_{\mu} b \bar{b} j+X$ at the LHC with $\sqrt{s}=8 \mathrm{TeV}$. The uncertainty bands depict the scale variation. The lower panel displays the differential $K$ factor and its uncertainty band. dashed (blue) curve corresponds to the LO, whereas the solid (red) one to the NLO result. The upper panels show the distributions themselves and the scale dependence bands that are constructed by calculating, bin-by-bin, a maximal and a minimal value out of the following set $\left\{m_{\mathrm{t}} / 2, m_{\mathrm{t}}, 2 m_{\mathrm{t}}\right\}$. The lower panels display the differential $\mathcal{K}$ factor. Higher order corrections to $p_{T j_{1}}$ do not simply rescale the shape of the LO distribution, but instead induce distortions. With a fixed scale choice they reach $-50 \%$ within the plotted range. Thus, the $p_{T j_{1}}$ differential cross section can only be properly described when the higher order corrections are taken into account. Therefore, LO calculations together with a suitably chosen global $\mathcal{K}$ factor would not approximate the full NLO QCD calculation well enough. However, a nearly constant $\mathcal{K}$ factor can be achieved with a judicious choice of the dynamic scale. Negative NLO corrections in the high $p_{T}$ tail means that the LO result is higher than the NLO one. The dynamic scale should depend on the $p_{T}$ of the hardest jet, and its value should increase in the tail of the distribution. On the other hand, the asymptotic freedom guarantees that the value of $\alpha_{s}$ becomes smaller there, resulting in lower NLO and LO cross sections. Because of the different dependence on the scale (see Figure 21), the LO cross section, which in general is much more sensitive to the variation of the scale, will change more rapidly than the NLO curve, driving a positive NLO/LO ratio in this region. We leave the search for such a scale for the future. On the contrary, for the $\Delta R_{e^{+} \mu^{-}}$distribution, negative, moderate and quite stable corrections are visible. This can be explained by the dimensionless nature of the observable. Certainly, $d \sigma / d \Delta R_{e^{+} \mu^{-}}$ receives contributions from all scales, most notably from those that are sensitive to the threshold for the $t \bar{t} j$ production. Indeed, for our scale choice, effects of the phase space regions close to this threshold dominate and a dynamic scale will not alter this behavior. Finally, the invariant mass distribution of the positron and $b$-jet is shown. In general, one cannot determine, which $b$-jet should be paired with the positron. To increase the probability that both final states come from the decay cascade initiated by the same top quark we select the $b e^{+}$pair, that returns the smallest invariant mass [38]. In case of the $t \bar{t}$ production this observable has proved to be particularly important for extracting $m_{\mathrm{t}}$ with a very high precision [39, 40. The top quark mass can be determined either from the shape of the distribution away from the kinematical endpoint, defined as $M_{b e^{+}}=\sqrt{m_{\mathrm{t}}^{2}-m_{\mathrm{W}}^{2}} \approx 153.5 \mathrm{GeV}$, or from the behavior of the observable in the vicinity of that point. In the former case, off-shell effects are negligible, in the latter they might even reach $50 \%$ 
41]. When the top quark and $W$ gauge boson decay on-shell, the end-point is represented by a sharp cut. However, additional radiation and off-shell effects introduce a smearing to the region, which is highly sensitive to the details of the description of the process. Thus, off-shell effects might prove to be very important for $t \bar{t} j$ as well, should top quark mass measurements be carried out using $M_{b e^{+}}$.

Summary and Outlook: In this Letter, NLO QCD corrections to $p p \rightarrow e^{+} \nu_{e} \mu^{-} \bar{\nu}_{\mu} b \bar{b} j+X$ with complete off-shell and interference effects have been calculated for the first time. We have shown that NLO QCD corrections to the total cross section are moderate $(13 \%)$. Nevertheless, their impact on some differential distributions is much larger. We have presented two cases, $p_{T j_{1}}$ and $M_{b e^{+}}$, where higher order corrections are indispensable to correctly describe the whole range of the observable. We have also estimated the size of the top quark off-shell effects at NLO for the total cross section, and confirmed that they are of the order of $\mathcal{O}\left(\Gamma_{\mathrm{t}} / m_{\mathrm{t}}\right)$. On the other hand, their influence on differential distributions might be much stronger, as has already been suggested by studies for the $p p \rightarrow e^{+} \nu_{e} \mu^{-} \bar{\nu}_{\mu} b \bar{b}$ production process [41]. We leave further comparisons for the future.

Acknowledgments: We would like to thank A. van Hameren for providing us with a new version of the KALEU software and C. G. Papadopoulos for useful discussions. The work of H. B. Hartanto was supported by the German Research Foundation (DFG) under Grant "Subtraction schemes at next-to-next-toleading order with applications to top-quark and jet physics". M. Worek and M. Kraus acknowledge support by the DFG under Grant No. WO 1900/1-1 - "Signals and Backgrounds Beyond Leading Order. Phenomenological studies for the LHC".

[1] S. Alioli, P. Fernandez, J. Fuster, A. Irles, S. O. Moch, P. Uwer and M. Vos, Eur. Phys. J. C 73 (2013) 2438, arXiv:1303.6415.

[2] D. L. Rainwater and D. Zeppenfeld, Phys. Rev. D 60 (1999) 113004 [Phys. Rev. D 61 (2000) 099901, hepph/9906218.

[3] N. Kauer, T. Plehn, D. L. Rainwater and D. Zeppenfeld, Phys. Lett. B 503 (2001) 113, hep-ph/0012351.

[4] M. L. Mangano, Eur. Phys. J. C 59 (2009) 373, arXiv:0809.1567.

[5] M. I. Gresham, I. W. Kim and K. M. Zurek, Phys. Rev. D 84 (2011) 034025, arXiv:1102.0018.

[6] S. Dittmaier, P. Uwer and S. Weinzierl, Phys. Rev. Lett. 98 (2007) 262002, hep-ph/0703120.

[7] S. Dittmaier, P. Uwer and S. Weinzierl, Eur. Phys. J. C 59 (2009) 625, arXiv:0810.0452.

[8] K. Melnikov and M. Schulze, Nucl. Phys. B 840
(2010) 129, arXiv:1004.3284.

[9] K. Melnikov, A. Scharf and M. Schulze, Phys. Rev. D 85 (2012) 054002, arXiv:1111.4991.

[10] A. Kardos, C. Papadopoulos and Z. Trocsanyi, Phys. Lett. B 705 (2011) 76, arXiv:1101.2672.

[11] S. Alioli, S. O. Moch and P. Uwer, JHEP 1201 (2012) 137, arXiv:1110.5251.

[12] M. Czakon, H. B. Hartanto, M. Kraus and M. Worek, JHEP 1506 (2015) 033, arXiv:1502.00925.

[13] A. Denner, S. Dittmaier, S. Kallweit and S. Pozzorini, Phys. Rev. Lett. 106 (2011) 052001, arXiv:1012.3975.

[14] G. Bevilacqua, M. Czakon, A. van Hameren, C. G. Papadopoulos and M. Worek, JHEP 1102 (2011) 083, arXiv:1012.4230.

[15] A. Denner, S. Dittmaier, S. Kallweit and S. Pozzorini, JHEP 1210 (2012) 110, arXiv:1207.5018.

[16] R. Frederix, Phys. Rev. Lett. 112 (2014) 8, 082002, arXiv:1311.4893.

[17] F. Cascioli, S. Kallweit, P. Maierhfer and S. Pozzorini, Eur. Phys. J. C 74 (2014) 3, 2783, arXiv:1312.0546.

[18] G. Heinrich, A. Maier, R. Nisius, J. Schlenk and J. Winter, JHEP 1406 (2014) 158, arXiv:1312.6659.

[19] A. Denner and R. Feger, arXiv:1506.07448 [hep-ph].

[20] G. Bevilacqua, M. Czakon, M. V. Garzelli, A. van Hameren, A. Kardos, C. G. Papadopoulos, R. Pittau and M. Worek, Comput. Phys. Commun. 184 (2013) 986, arXiv:1110.1499.

[21] A. van Hameren, C. G. Papadopoulos and R. Pittau, JHEP 0909 (2009) 106, arXiv:0903.4665.

[22] G. Ossola, C. G. Papadopoulos and R. Pittau, JHEP 0803 (2008) 042, arXiv:0711.3596.

[23] G. Ossola, C. G. Papadopoulos and R. Pittau, Nucl. Phys. B 763 (2007) 147, hep-ph/0609007.

[24] G. Ossola, C. G. Papadopoulos and R. Pittau, JHEP 0805 (2008) 004, arXiv:0802.1876.

[25] P. Draggiotis, M. V. Garzelli, C. G. Papadopoulos and R. Pittau, JHEP 0904 (2009) 072, arXiv:0903.0356.

[26] A. Denner, S. Dittmaier, M. Roth and D. Wackeroth, Nucl. Phys. B 560 (1999) 33, hep-ph/9904472.

[27] A. Denner, S. Dittmaier, M. Roth and L. H. Wieders, Nucl. Phys. B 724 (2005) 247 [Nucl. Phys. B 854 (2012) 504], hep-ph/0505042.

[28] A. van Hameren, Comput. Phys. Commun. 182 (2011) 2427, arXiv:1007.4716.

[29] S. Catani and M. H. Seymour, Nucl. Phys. B 485 (1997) 291 [Nucl. Phys. B 510 (1998) 503], hepph/9605323.

[30] S. Catani, S. Dittmaier, M. H. Seymour and Z. Trocsanyi, Nucl. Phys. B 627 (2002) 189, hep-ph/0201036.

[31] M. Czakon, C. G. Papadopoulos and M. Worek, JHEP 0908 (2009) 085, arXiv:0905.0883.

[32] G. Bevilacqua, M. Czakon, M. Kubocz and M. Worek, JHEP 1310 (2013) 204, arXiv:1308.5605.

[33] C. G. Papadopoulos, Comput. Phys. Commun. 137 (2001) 247, hep-ph/0007335.

[34] A. van Hameren, arXiv:1003.4953.

[35] M. Jezabek and J. H. Kuhn, Nucl. Phys. B 314 (1989) 1 .

[36] A. D. Martin, W. J. Stirling, R. S. Thorne and G. Watt, Eur. Phys. J. C 63 (2009) 189, arXiv:0901.0002.

[37] M. Cacciari, G. P. Salam and G. Soyez, JHEP 0804 
(2008) 063, arXiv:0802.1189.

[38] M. Beneke et al., hep-ph/0003033.

[39] S. Chatrchyan et al. [CMS Collaboration], Eur. Phys. J. C 73 (2013) 2494, arXiv:1304.5783.
[40] G. Aad et al. [ATLAS Collaboration], Eur. Phys. J. C 75 (2015) 7, 330, arXiv:1503.05427.

[41] J. Alcaraz Maestre et al., arXiv:1203.6803. 